\title{
Deep Convolution Neural Networks Learned Image Classification for Early Cancer Detection using Lightweight
}

\section{Dr. K Seshadri Ramana ${ }^{a}$, K. Bala Chowdappa ${ }^{b}$, Dr. O. Obulesu ${ }^{c}$, Dr.Deena Babu Mandru ${ }^{\mathrm{d}}$ and Dr.Suresh Kallam ${ }^{\mathrm{e} *}$}

\footnotetext{
${ }^{a}$ Professor,Department of Computer Science and Engineering,G. Pullaiah College of Engineering and Technology (Autonomous), Kurnool ,AP,India-518002.

${ }^{\mathrm{b}}$ Assistant Professor,Department of Computer Science and Engineering, G Pulla Reddy Engineering College (Autonomous), Kurnool ,AP,India-518007.

${ }^{\mathrm{c}}$ Associate Professor, Department of IT, G.Narayanamma Institute of Technology \& Science (Autonomous), Shaikpet, Hyderabad,India- 500104.

${ }^{\mathrm{d}}$ Professor \& HoD, Department of IT, Malla Reddy Engineering College(Autonomous), Maisammaguda, Secunderabad,India-500100.

${ }^{*}$ Associate Professor, Department of Computer Science and Engineering, SreeVidyanikethan Engineering College (Autonomous), Tirupati ,AP,India-517126.
}

Corresponding Author email:sureshkallam@gmail.com*

\begin{abstract}
Cancer is uncontrolled cell growth in any part of the body. Early cancer detection aims to identify patients who exhibit symptoms early on in order to maximise their chances of a successful treatment. Cancer disease mortality is decreased through early detection and treatment. Numerous researchers proposed a variety of image processing and machine learning approaches for cancer detection. However, existing systems did not improve detection accuracy or efficiency. A Deep Convolutional Neural Learning Classifier Model based on the Least Mean Square Filterative Ricker Wavelet Transform (L-DCNLC) is proposed to address the aforementioned issues. The L-DCNLC Model's primary objective is to detect cancer earlier by utilising a fully connected max pooling deep convolutional network with increased accuracy and reduced time consumption. The fully connected max pooling deep convolutional network is composed of one input layer, three hidden layers, and one output layer. Initially, the input layer of the L-DCNLC Model considers the number of patient images in the database as input.
\end{abstract}


Following that, preprocessing is performed in hidden layer 1 to perform denoising in order to improve image quality. This is accomplished by employing the Least Mean Square Weiner Filtering process, which has a higher peak signal-to-noise ratio. The Continuous Ricker Wavelet Transform is then used to extract the image features from the preprocessed image in hidden layer 2. Finally, the classification process is performed in hidden layer 3 using the Kulczynksi Similarity Coefficient to detect the cancerous image by comparing the testing and extracted features. Thus, the cancerous image is detected and displayed with a low error rate in the output layer. The brain cancer and lung cancer datasets are analysed to determine peak signal to noise ratios (PSNR), cancer detection accuracy, and cancer detection time. The evaluation results indicate that the L-DCNLC Model improves accuracy and PSNR while requiring less computational time than previous works.

\section{Keywords: machine learning, classification, image processing, Cancer detection, continuous ricker wavelet transform.}

\section{INTRODUCTION}

Cancer is a common disease that kills people worldwide, according to the World Health Organization (WHO). Early detection of cancer saves lives throughout the world. Due to the shape variation and cancer area, developing computer-aided detection systems is a difficult task that has remained an open problem. Numerous methods for detecting brain cancer using MR and CT images were reviewed, as well as the benefits and drawbacks of detecting brain and lung cancer. [1,] used a discrete orthonormal S-transform (DOST) and principal component analysis (PCA) to mine texture features, and linear discriminant analysis was used to reduce dimensionality (LDA). The Adaboost algorithm with random forest (ADBRF) classifier was used to perform classification. On the other hand, did not impair cancer detection accuracy. [2,] developed a deep learning framework for detecting prostate cancer in CEUS images. Convolution was used to extract features from temporal dimensions for cancer detection. The deep learning framework, on the other hand, did not result in a reduction in the time required to detect cancer. [3] introduced deep wavelet autoencoder (DWA) image compression with image decomposition and feature reduction. Combining the two significantly reduced the sinking 
feature set size for DNN classification tasks. DWA had no effect on classification accuracy, however. [4] developed a region growing algorithm based on fuzzy theory and a region growing algorithm. The algorithm developed was used to partition images and enhance segmentation. However, the algorithm for growing regions did not result in a reduction in the time required to detect cancer. [5,] introduced an optimised deep learning mechanism called Dolphin-SCA-based Deep $\mathrm{CNN}$ to increase accuracy through effective classification decisions. The feature extraction process was guided by statistical features. While the accuracy was increased, the feature extraction took an inordinate amount of time. Convolutional neural networks (CNNs) were introduced in [6] for feature extraction and classification. To increase the success rate of classification, image augmentation techniques such as cutting and filling were introduced. CNN, on the other hand, did not improve the error rate. [7] introduced a deep learning model for categorising dermal cell images and detecting skin cancer. A model-driven architecture based on deep learning algorithms was developed to predict skin cancer. The deep learning model, on the other hand, did not reduce computational complexity. [8] introduced a hybrid feature extraction method for cancer categorization. Preprocessing was used to enhance the quality of the designed approach. However, the feature extraction technique was not used to perform the image denoising. [9] developed the Artificial Bee Colony Algorithm for the selection and classification of cancer features. The classification time, on the other hand, was not shortened. [10] introduced a weighted mean histogram equalisation approach for the purpose of removing noise and improving image quality. A clustering technique was used to segment the infected region. The weighted mean histogram equalisation method, on the other hand, had no effect on the peak signal-to-noise ratio.

The disadvantages of the existing cancer detection methods discussed previously include a lower peak signal-to-noise ratio, decreased accuracy, increased time, increased computational cost, and increased computational complexity. No advanced detection technique was used to detect the cancer disease at the outset. To address the aforementioned issues, the L-DCNLC Model is introduced.

The paper is divided into five distinct sections. The proposed L-DCNLC Model is briefly described in section 2 with different cancer detection stages. The third section discusses related works in cancer detection techniques. Section 4 presents experimental settings along with a 
detailed database explanation. Section 5 analyses the results for three different parameters. Section 6 contains the conclusion.

\section{RELATED WORKS}

[11] described the use of an extreme learning machine algorithm for detecting brain tumours in MR images of the brain. The computational cost, on the other hand, was not reduced. The Berkeley wavelet transformation (BWT) was developed in [12] with the goal of reducing complexity while increasing accuracy. To extract relevant features from the segmented area, a support vector machine classifier was used. While accuracy was increased, time consumption did not decrease.

The article [13] described an automated method for detecting and segmenting abnormal tissue using Magnetic Resonance Imaging. The fully automated method, on the other hand, did not result in a reduction in time consumption. [14] described a novel hybrid energy-efficient method for tumour detection and segmentation. The Fuzzy C-Means method was used to segment tumours. However, the hybrid energy-efficient method had no effect on the error rate.

[15] proposed an alternating direction method to address clinical diagnosis issues. To improve accuracy, the method was designed to reflect both the global structure of the image and the local pixel information. However, the computational complexity was not reduced. [16] introduced a novel two-phase multi-model for tumour detection. The developed model was used to classify MRI images as normal or abnormal in order to identify the brain tumour. The two-phase multimodel, on the other hand, did not improve classification accuracy. A Tripartite Generative Adversarial Network (Tripartite-GAN) was introduced in [17] to identify the tumour without using the CA injection. The Tripartite-GAN model, on the other hand, did not reduce the error rate. [18] introduced a new computer-aided detection (CADe) system with the goal of decreasing clinician reading time and increasing efficiency. To identify breast tumour candidates, a phasebased approach was used on a local level. However, the time complexity was overlooked. In [19], CNN, a deep learning network, was introduced for diagnosing brain tumours using MRI images. However, the planned network did not complete the pre-processing task. [20] introduced a method for noise removal that included feature extraction and DWT-based segmentation to 
reduce complexity. However, the developed method did not improve the accuracy of cancer detection.

\section{METHODOLOGY}

Image processing is the process of performing operations on an input image in order to improve it by extracting important information. Early cancer detection allows for the identification of the tumour and subsequent treatment. Many researchers conducted their studies in order to detect cancer at an earlier stage. However, neither the detection accuracy nor the time consumption were improved. To address these issues, the Least Mean Square Filterative Ricker Wavelet Transform based Deep Convolutional Neural Learning Classifier (L-DCNLC) Model is presented. The primary goal of the L-DCNLC Model is to detect cancer with greater accuracy and in less time. Figure 1 depicts the architecture diagram of the L-DCNLC Model.

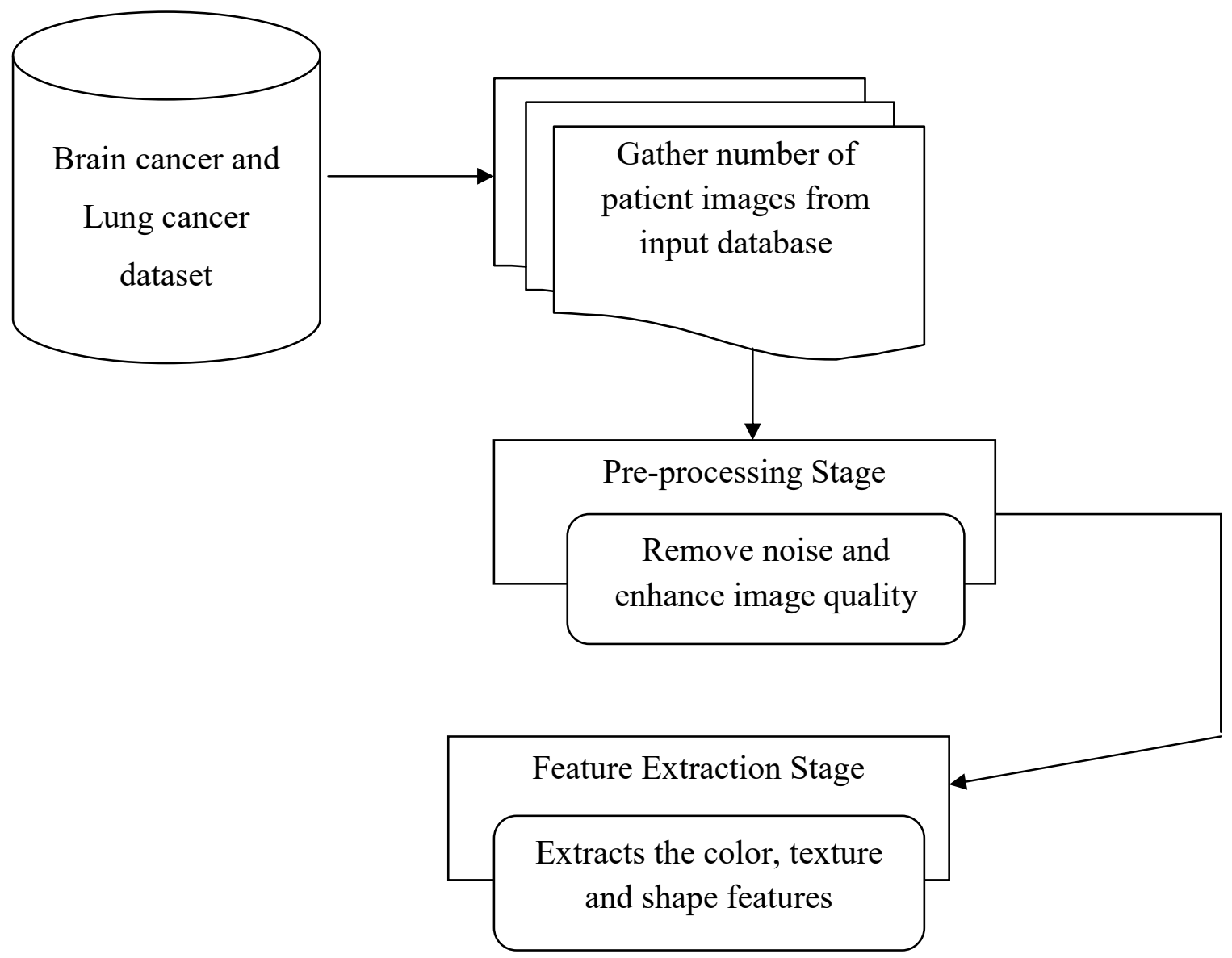




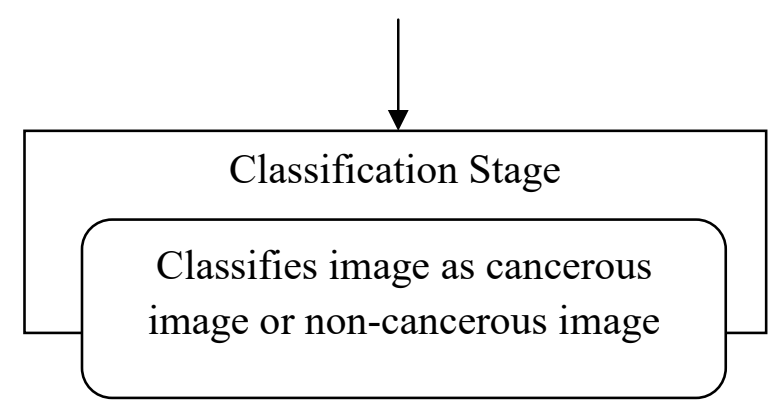

\section{Figure 1 Structural design of the proposed L-DCNLC Model}

Figure 1 depicts the proposed L-DCNLC Model design. Images are first extracted from the input dataset. The image quality was then improved by preprocessing by removing noisy pixels using the Least Mean Square Weiner Filtering process. The image features are then extracted using the Continuous Ricker Wavelet Transform. Finally, the classification process is used to identify the cancerous image. The following subsection describes the detailed process of the L-DCNLC Model.

\subsection{Least Squares Filterative Deep Convolutional Neural Learning Classifier Based on Ricker} Wavelet Transform

The L-DCNLC Model employs a fully connected max pooling deep convolutional network to detect cancer accurately. The network is designed with a cascade of processing layers for preprocessing, feature extraction, and classification. Every subsequent layer builds on the previous layer's input. One input layer, three hidden layers, and one output layer comprise the fully connected max pooling deep convolutional network. The fully connected max pooling network learns the input images in the hidden layers and transforms them into an output layer in a feed-forward fashion. Figure 2 depicts a fully connected max pooling deep convolutional network.

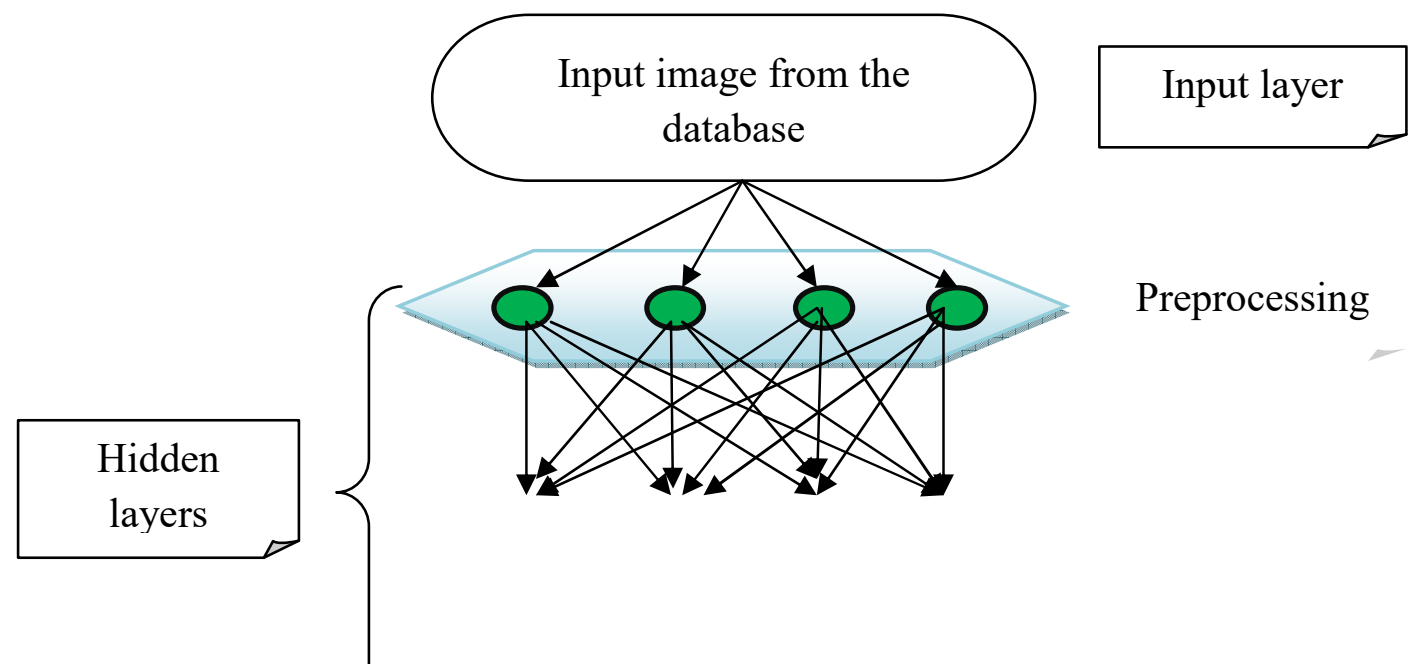




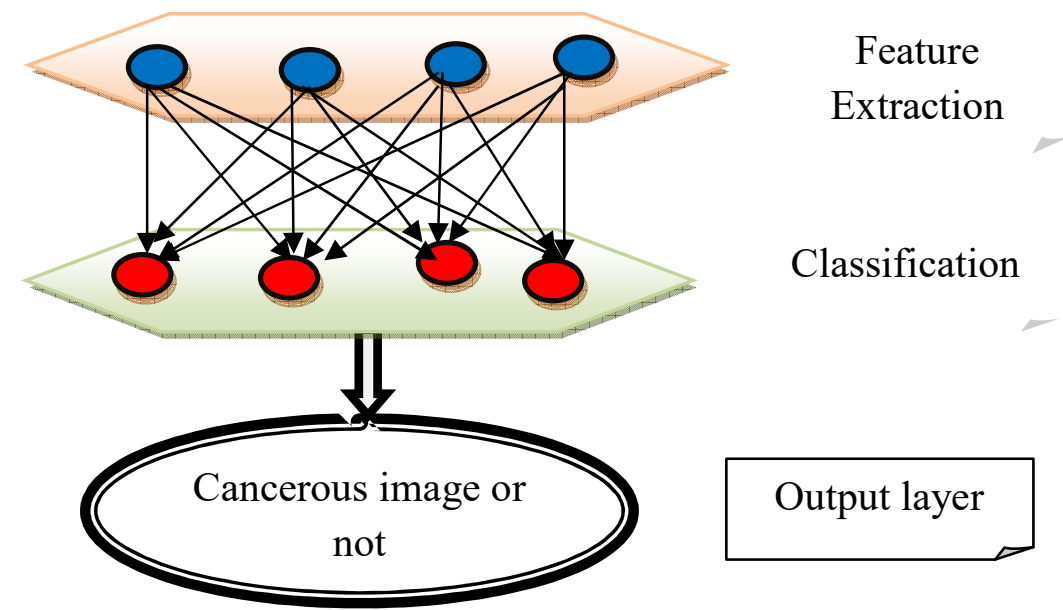

Figure 2 Diagrammatic representation of fully connected max pooling deep convolution network

The diagrammatic representation of fully connected max pooling deep convolutional network is shown in figure 2. In designed network, neurons like nodes are linked to other layers to form an entire network. The input layer comprises the image from the database and formulated as,

$$
\operatorname{Input}(t)=\sum_{i=1}^{n} I_{i}(t) \varphi_{i h}+d
$$

In (1), 'Input(t)' denotes an input at a specific time instant.

' $t$ ', 'I i (t)' denotes the input from the dataset, and ' the hidden layer. The letter ' $d$ ' represents bias. The input layer then sends the collected inputs to the hidden layer 1. Image preprocessing is done in this layer. The proposed L-DCNLC Model begins with preprocessing.

The database is used to determine the number of images. Normally, the original images are harmed by unwanted noise, which reduces image quality. The presence of noise in the input image increases the complexity of cancer detection. As a result, preprocessing is critical for improving image quality and reducing noisy pixels. For input image pre-processing, the proposed L-DCNLC Model employs the Least Mean Square Weiner Filtering (LMSWF) process. The total number of images collected is ' $\mathrm{I}$ 1,I 2,I 3...,I $\mathrm{n}$ '. LMSWF is a stochastic and 
statistical method for noise removal that employs inverse filtering and noise smoothing. The blur and noise in the input image are reduced by LMSWF. The principle of orthogonality includes In the Fourier domain, the Wiener filter is defined as

$$
W F(u, v)=\frac{B(u, v) P_{y y}(u, v)}{|B(u, v)|^{2} P_{y y}(u, v)+P_{z z}(u, v)}
$$

The power spectra of the input image and noise are denoted by 'P yy $(\mathrm{u}, \mathrm{v})$ ' and ' $\mathrm{P}(\mathrm{zz})$ $(\mathrm{u}, \mathrm{v})$ ' in (2). The blurring filter is denoted by 'B(u,v),' and the Weiner filter is denoted by 'WF(u,v).' The preprocessed image is obtained in this manner, and thus the PSNR improves.

The preprocessed image is then sent to hidden layer 2. The feature extraction process is carried out in that layer to reduce the dimension in order to provide the interesting parts of an image.

The L-DCNLC Model decomposes the preprocessed image and generates different subblocks for horizontal and vertical directions using the Continuous Ricker Wavelet Transform (CRWT). Figure 3 depicts an example of an input pre-processed image.
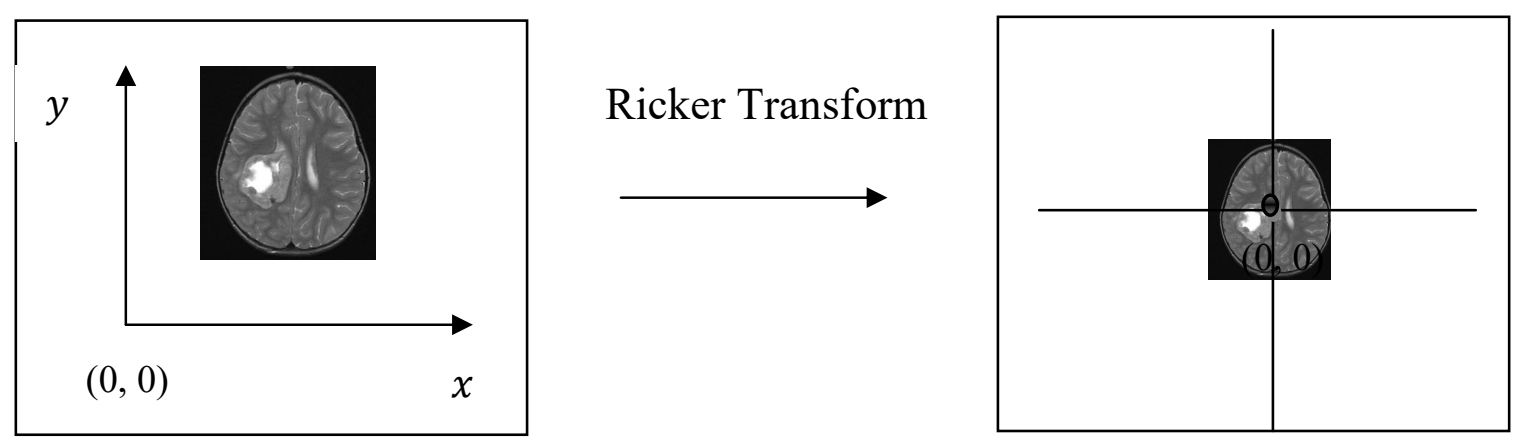

Figure 3 Ricker transform

Figure 3 describes the Ricker transform of input image. Then, the pre-processed input image is converted into single point in parameter space.

\section{PERFORMANCE DISCUSSION}

The qualitative and quantitative performance analysis is carried out in this section.

The first subsection explains qualitative results analysis. The quantitative analysis is carried out using various metrics, which are discussed in the second sub-section.

\subsection{Qualitative performance analysis}


A qualitative analysis of the proposed L-DCNLC model is performed using two different datasets, one for brain cancer and one for lung cancer.Initially, the input MRI and CT images are obtained from the database.

\begin{tabular}{|c|c|c|}
\hline Process & Brain cancer dataset & Lung cancer dataset \\
\hline Input image & & \\
\hline $\begin{array}{l}\text { Least Mean Squa } \\
\text { Weiner Filter bas } \\
\text { Image preprocess }\end{array}$ & & \\
\hline Texture featur & & \\
\hline Color feature & & $\frac{1}{4}$ \\
\hline
\end{tabular}




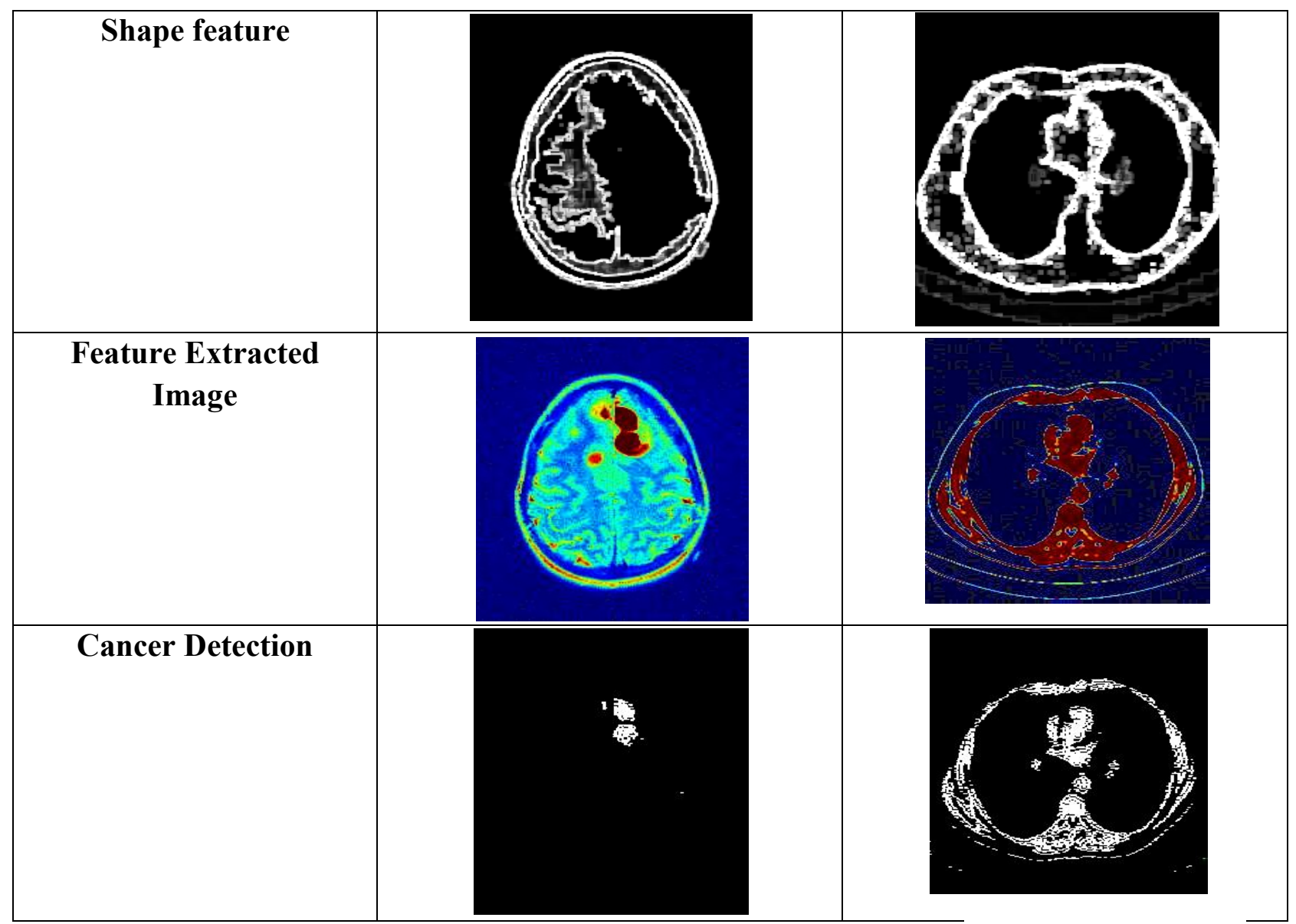

Figure 4 Qualitative performance analysis of L-DCNLC model for Brain cancer dataset and Lung cancer dataset

The quality results for the L-DCNLC model are shown in Figure 4. The MRI and CT images original input are taken from the database. In order to obtain a better quality picture, the least mean square weiner-based image preprocessing is performed. The image is then removed with the texture, shape and colour features. Finally, the Kulczynksi Similarity Coefficient is used to detect the cancer.

\subsubsection{Cancer detection time}

Cancer detection time is the time consumed by the technique to detect the normal or cancerous image. The cancer detection time is determined as,

$$
C D_{\text {Time }}=n * \text { Time }(\text { detecting one image })(14)
$$


From (14), ' $C D_{\text {Time }}$ ' represents the cancer detection time and ' $n$ ' denotes the number of input images. The cancer detection time is computed in milliseconds (ms).

Table 1 Tabulation of cancer detection time for brain cancer dataset

\begin{tabular}{|c|c|c|c|}
\hline $\begin{array}{c}\text { number of } \\
\text { input } \\
\text { images }\end{array}$ & L-DCNL & DOST+PCA+LDA+ADBRF & $\begin{array}{c}\text { Deep } \\
\text { Learning } \\
\text { frame work }\end{array}$ \\
\hline 25 & 7.5 & 11.9 & 16.25 \\
\hline 50 & 16.97 & 15.5 & 32.5 \\
\hline 75 & 28 & 40 & 51.2 \\
\hline 100 & 38 & 54 & 69 \\
\hline 125 & 47.45 & 67.5 & 88 \\
\hline 150 & 59.9 & 86 & 107.5 \\
\hline 175 & 72.9 & 102 & 130 \\
\hline 200 & 86.5 & 121 & 150 \\
\hline
\end{tabular}

Figure 3 shows how the number of images calculated is used to analyse cancer detection time. The line graph in the image above depicts a patient's diagnosis date for brain cancer versus lung cancer. According to the three different conventional methods demonstrated above, the LDCNLC model has the cancer detection time that is better. Because a fully connected max pooling deep convolutional network was trained using the L-DCNLC model, this is the result. Before processing, noise was removed. In addition to the colour, texture, and shape data that remains, it also includes colour, texture, and shape information. After classifying the image, the results show whether it is cancerous. Consequently, this helps to speed up the cancer detection process. The cancer detection time of the L-DCNLC model is reduced by $30 \%$ and $44 \%$ when compared to the current DOST $+\mathrm{PCA}+\mathrm{LDA}+\mathrm{ADBRF}$ [1] and Deep learning framework [2]. When comparing L-DCNLC [1] and Deep learning framework [2], the lung cancer detection time of L-DCNLC [1] is $22 \%$ slower and Deep learning framework [2] is $33 \%$ slower. 


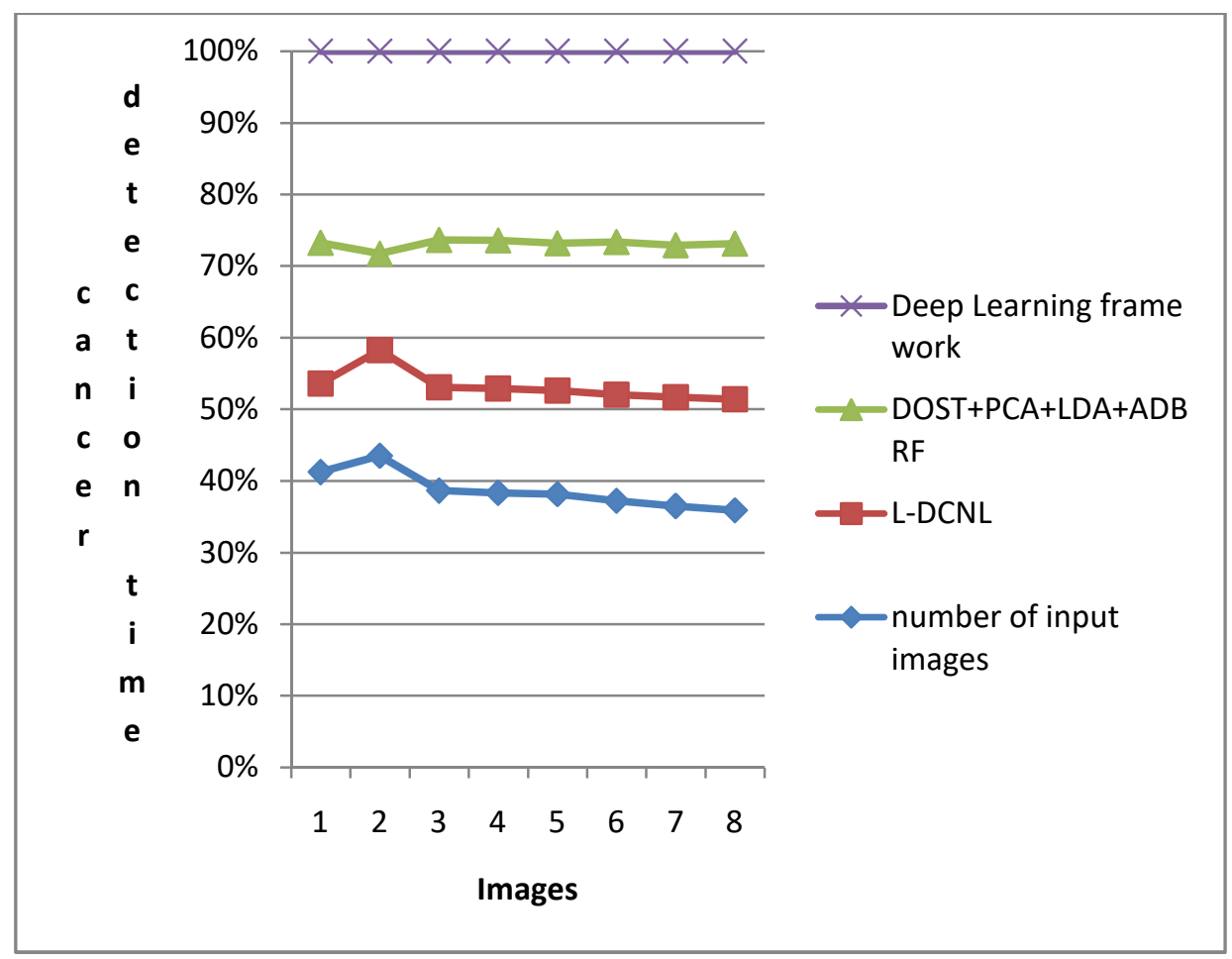

Figure 4 Measurement of cancer detection time

In figure 4 , we see that the calculation of the number of images is used to analyse cancer detection time. In the above graph, the red line represents the time when a patient was diagnosed with brain cancer, and the green line represents the time when a patient was diagnosed with lung cancer. L-DCNLC model's cancer detection time is better than the other two conventional methods demonstrated above. This is because the L-DCNLC model was used to train a fully connected max pooling deep convolutional network. The image quality was improved by removing noise before processing. And the remaining data after that includes colour, texture, and shape information. Following that, the image is classified to determine if it is cancerous. As a result, this helps to shorten the time needed to detect cancer. Compared to existing DOST $+\mathrm{PCA}+\mathrm{LDA}+\mathrm{ADBRF}$ [1] and Deep learning framework [2], the average cancer detection time of L-DCNLC model is reduced by $30 \%$ and $44 \%$. The lung cancer detection time of LDCNLC [1] and Deep learning framework [2] are 22\% and 33\% respectively, when compared to existing DOST+PCA+LDA+ADBRF [2]. 


\section{CONCLUSION}

The L-DCNLC model is introduced in this paper, along with three distinct processes: preprocessing, feature extraction, and classification. Initially, the L-DCNLC model removes noise from the input image in order to obtain a high-quality image for accurate classification. Following that, the significant image features are extracted from the preprocessed image in order to reduce the time required for cancer detection. Finally, the similarity coefficient is used to perform the classification process in order to detect cancer based on the extracted features with greater accuracy and a lower error rate. The extensive experimental evaluation is carried out using an MRI brain image and a CT lung image database. The qualitative and quantitative analysis is carried out in the section titled "results and discussion." When compared to other related works, the quantitative results are verified in terms of higher cancer detection accuracy and shorter time.

\section{Declaration:}

Author Contribution: All authors have equally contributed and all authors have read agreed to the published version of the manuscript.

Funding: This research has no funding by any organization or individual.

Institutational review board statement: Not applicable.

Informed consent statement: Not applicable.

Data availability statement: Data sharing not applicable.

Conflicts of interest: The authors declare that they have no conflicts of interest.

\section{REFERENCES}

[1] Sonali Mishra, Banshidhar Majhi and Pankaj Kumar Sa, "Texture feature based classification on microscopic blood smear for acute lymphoblastic leukemia detection", Biomedical Signal Processing and Control, Elsevier, Volume 47, 2019, Pages 303-311 
[2] Yujie Feng, Fan Yang, Xichuan Zhou, Yanli Guo, Fang Tang, Fengbo Ren, Jishun Guo and Shuiwang Ji, "A Deep Learning Approach for Targeted Contrast-Enhanced Ultrasound Based Prostate Cancer Detection", IEEE/ACM Transactions on Computational Biology and Bioinformatics, Volume 16, Issue 6, 2019, Pages 1-8

[3] Pradeep Kumar Mallick, Seuc Ho Ryu, Sandeep Kumar Satapathy, Shruti Mishra, Gia Nhu Nguyen and Prayag Tiwari, "Brain MRI Image Classification for Cancer Detection Using Deep Wavelet Autoencoder-Based Deep Neural Network", IEEE Access, Volume 7, Pages 46278 46287

[4] Gongfa Li, Du Jiang, Yanling Zhou, Guozhang Jiang, Jianyi Kong and Gunasekaran Manogaran, "Human Lesion Detection Method Based on Image Information and Brain Signal", IEEE Access, Volume 7, Pages 11533 - 11542

[5] Sharan Kumar, DattatreyaP.Mankamem, "Optimization driven Deep Convolution Neural Network for brain tumor classification", Biocybernetics and Biomedical Engineering, Elsevier, Volume 40, Issue 3, 2020, Pages 1190-1204

[6] Mesut Togacar, Burhan Ergen and Zafer Comert, "Detection of lung cancer on chest CT images using minimum redundancy maximum relevance feature selection method with convolutional neural networks", Biocybernetics and Biomedical Engineering, Elsevier, Volume 40, Issue 1, January-March 2020, Pages 23-39

[7] Mohammad Ali and Kadampur Sulaiman Al Riyaee, "Skin cancer detection: Applying a deep learning based model driven architecture in the cloud for classifying dermal cell images", Informatics in Medicine Unlocked, Elsevier, Volume 18, 2020

[8] Abdu Gumaei, Mohammad Mehedi Hassan, Md Rafiul Hassan, Abdulhameed Alelaiwi and Giancarlo Fortino, “A Hybrid Feature Extraction Method with Regularized Extreme Learning Machine for Brain Tumor Classification”, IEEE Access, Volume 7, March 2019, Pages 36266 36273

[9] B. Karthiga and M. Rekha, "Feature extraction and I-NB classification of CT images for early lung cancer detection”, Materias today, Elsevier, June 2020, Pages 1-8 
[10] P. Mohamed Shakeel, M. A. Burhanuddin and Mohamad Ishak Desa, "Lung cancer detection from CT image using improved profuse clustering and deep learning instantaneously trained neural networks”, Measurement, Elsevier, Volume 145, October 2019, Pages 702-712

[11] Fatih Özyurt, Eser Sert and Derya Avc1, "An expert system for brain tumor detection: Fuzzy C-means with superresolution and convolutional neural network with extreme learning machine", Medical Hypotheses, Elsevier, Volume 134, 2020, Pages 1-8

[12] Nilesh BhaskarraoBahadure, Arun Kumar Ray, and Har Pal Thethi, "Image Analysis for MRI Based Brain Tumor Detection and Feature Extraction Using Biologically Inspired BWT and SVM", International Journal of Biomedical Imaging, Hindawi, Volume 2017, March 2017, Pages 1-12

[13] Mohammadreza Soltaninejad, Guang Yang, Tryphon Lambrou, Nigel Allinson, Timothy L. Jones, Thomas R. Barrick, Franklyn A. Howe and Xujiong Ye, "Automated brain tumour detection and segmentation using superpixel-based extremely randomized trees in FLAIR MRI", International Journal of Computer Assisted Radiology and Surgery, Springer, Volume 12,2017, Pages 183-203

[14] P. G. Rajan and C. Sundar, "Brain Tumor Detection and Segmentation by Intensity Adjustment", Journal of Medical Systems, Springer, Volume 43, Issue 282, 2019, Pages 1-13

[15] Ting Ge, Ning Mu, Tianming Zhan, Zhi Chen, Wanrong Gao and Shanxiang Mu, "Brain Lesion Segmentation Based on Joint Constraints of Low-Rank Representation and Sparse Representation", Computational Intelligence and Neuroscience, Hindawi, Volume 2019, July 2019, Pages 1-11

[16] Mahmoud Khaled Abd-Ellah, Ali Ismail Awad, Ashraf A. M. Khalaf and Hesham F. A. Hamed, "Two-phase multi-model automatic brain tumour diagnosis system from magnetic resonance images using convolutional neural networks", EURASIP Journal on Image and Video Processing, Springer, Volume 2018, Issue 97, 2018, Pages 1-10

[17] Jianfeng Zhao, Dengwang Li , Zahra Kassam, Joanne Howey, Jaron Chong, Bo Chen and Shuo Li, "Tripartite-GAN: Synthesizing liver contrast-enhanced MRI to improve tumor detection", Medical Image Analysis, Volume 63, 2020, Pages 1-16 
[18] Xin Wang, Yi Guo, Yuanyuan Wang and Jinhua Yu, "Automatic breast tumor detection in ABVS images based on convolutional neural network and superpixel patterns", Neural Computing and Applications, Springer, Volume 31, 2019, Pages1069-1081

[19] Ahmet Cinar and Muhammed Yildirim, "Detection of tumors on brain MRI images using the hybrid convolutional neural network architecture", Medical Hypotheses, Elsevier, Volume 139, June 2020, Pages 1-8

[20] N. Varuna Shree and T. N. R. Kumar, "Identification and classification of brain tumor MRI images with feature extraction using DWT and probabilistic neural network", Brain Informatics, Springer, Volume 5, 2018, Pages 23-30 